\title{
Microfluidic paper-based analytical devices: from POCKET to paper-based ELISA
}

\author{
4 years after the initial publication on microPADs, the simple idea of making microfluidic devices out of paper is \\ being adopted by a growing number of researchers."
}

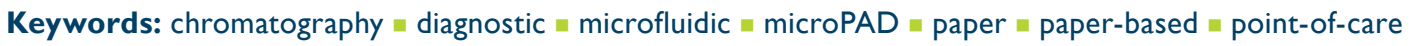

Microfluidic paper-based analytical devices (microPADs) began as a simple idea with an ambitious goal. The idea was to make microfluidic devices out of paper instead of plastic or glass. The goal was to develop low-cost and portable paper-based diagnostic devices to improve healthcare in developing countries. Over the past 6 years, many developments have been made in the emerging field of paper-based microfluidic devices. Reviewing the development of these devices in the Whitesides group at Harvard University (Cambridge, MA, USA) can provide some insight into the future of the field and encourage scientists from a variety of backgrounds to contribute their expertise toward further development of useful microPADs.

\section{POCKET to paper}

In 2004, the Whitesides group introduced POCKET, a portable microfluidic-based system designed to address the need for simple diagnostic devices in developing countries [1]. POCKET could run quantitative immunoassays in the field with the same sensitivity as bench-top ELISA, but in a fraction of the time and at a fraction of the cost. This project was the culmination of years of work with conventional microfluidic devices made from poly(dimethylsiloxane) and marked the shift towards developing microfluidic devices out of paper [2].

The inspiration to work with paper came from the belief that conventional microfluidic devices were too complicated and expensive to be used on a large scale in developing countries. Paper was selected as the material for making a new generation of simple microfluidic devices because paper is cheap and has the ability to wick fluids by capillary action, thus no pumps or power sources would be required to move fluids through a device made out of paper. In addition to these two important characteristics, paper also has a high surface-to-volume ratio, which is ideal for assays where reagents are bound to the surface of the paper; white paper provides an excellent background for colorimetric assays; paper is flammable, so devices made out of paper can be easily disposed of by incineration; and paper has been used extensively as a platform for assays. The most widely used point-of-care diagnostic devices are paper-based dipsticks, such as urinalysis dipsticks and lateral-flow immunoassays, such as pregnancy tests [3]. All of these assays can be adapted to microPADs.

However, paper also has disadvantages as a material for diagnostic devices: it is not well suited for absorbance measurements because the paper fibers lead to large amounts of light scattering [4], the wicking rate of paper is not always perfectly uniform even within a single sheet of paper, and the distance a fluid will wick along a channel in paper is proportional to the square root of time, so fluids can wick across short channels $(<3 \mathrm{~cm})$ in minutes, but, depending on the humidity and channel dimensions, may require hours to wick across long channels $(>5 \mathrm{~cm})$ [5].

Paper is a term used to describe thin sheets made typically from pressed cellulose fibers. For developing microfluidic devices, it is convenient to use the term 'paper' more broadly to include any material in the form of a thin $(\leq 1 \mathrm{~mm})$ and flexible sheet that wicks aqueous solutions by capillary action. This broader definition of paper includes thousands of commercially available materials, ranging from everyday paper towels to highly engineered polymer membranes with a wide variety of wicking rates, thicknesses, pore sizes, chemical compositions and costs. When selecting the paper to make a microPAD, one must consider the properties of the paper since they translate directly into the properties of the channels in the device.

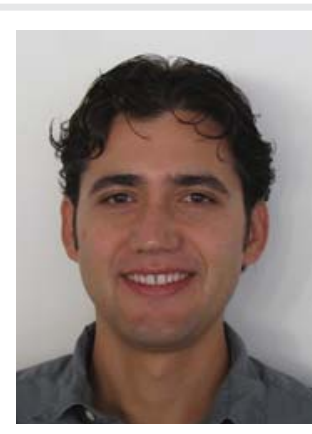

Andres W Martinez

Department of Chemistry Biochemistry, California Polytechnic State University, I Grand Avenue,

San Luis Obispo, CA 93407, USA

Tel.: + I 8057562744

Fax: +l 8057565500

E-mail:awmartin@calpoly.edu 


\section{Patterning paper}

To make a microPAD, it is necessary to pattern paper into regions of hydrophilic paper (e.g., channels that wick fluids) bounded by regions of hydrophobic paper (barriers). Initial attempts to pattern paper involved stamping patterns of Scotchguard ${ }^{\mathrm{TM}}$ on paper, but when the stamp was applied to a sheet of paper, the liquid Scotchguard wicked in all directions on the paper, blurring the pattern beyond recognition. After testing dozens of waterproofing solutions, polymers and waxes, a method for patterning paper based on photolithography was developed, and the first paper-based microfluidic device was introduced in 2007 [6]. Within a year, two additional methods for patterning paper were published by other groups $[7,8]$. There are presently seven methods for patterning paper: photolithography, plotting, inkjet printing, plasma etching, flexographic printing [9], wax printing and cutting patterns of channels from a sheet of paper [10]. Devices made by cutting are unique, in that the paper channels are bounded by air instead of hydrophobic paper [11]. Innovative methods for patterning paper are always welcome in the field, as each new method for patterning paper brings new capabilities to the devices.

\section{प्रा \\ "The interest in working with paper came from the belief that conventional microfluidic devices were too complicated and expensive to be used on a large scale in developing countries."}

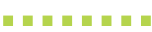

Wax printing and cutting are currently the most convenient methods for patterning paper. In wax printing, a pattern of wax is printed on a sheet of paper using a commercially available solid-ink printer. The paper is then heated to melt the wax so that it spreads through the thickness of the paper, thus creating a hydrophobic barrier. The method is simple to learn, rapid ( $\sim 5 \mathrm{~min})$ and only requires two pieces of equipment - a solid-ink printer and a hot plate or oven [12]. Cutting is also simple, rapid and only requires a cutting instrument such as a pair of scissors or, for more complex patterns, a laser cutter.

\section{Applications of microPADs: paper-based ELISA \& beyond}

A large portion of the initial work with microPADs was focused on developing methods of fabricating devices and optimizing components in the devices, such as channels, test zones, sample inlets, valves, diluters and mixers [10]. To demonstrate some of the applications of microPADs, colorimetric assays for several analytes including glucose, bovine serum albumin, nitrites, ketones, alkaline phosphatase and cholesterol were demonstrated [10]. These simple tests illustrated the qualities of paper-based devices: the devices were small, inexpensive, easy to use, required small volumes of sample and could provide quantitative results when combined with a detector such as a camera phone. Several other detection methods have also been combined with paper-based devices including electrochemical detection [10], transmittance detection [4], chemiluminescence detection [13] and fluorescence detection [14].

\section{"MicroPADs have still not been developed into a diagnostic system comparable to POCKET, but recent work on paper-based ELISA represents a step toward this goal."

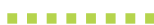

While there are still many opportunities for developing new components for paper-based devices, microPADs have advanced to the point where researchers can now focus on developing working assays that can be used in the field for diagnosing disease or detecting analytes. MicroPADs have still not been developed into a diagnostic system comparable to POCKET, but recent work on paper-based ELISA represents a step toward this goal [14]. Paper-based ELISA demonstrated that conventional ELISA could be performed on patterned paper. Paper-based ELISA is faster and less expensive than conventional bench-top ELISA performed in a 96-well plate, but the sensitivity of paper-based ELISA is currently more than ten-times lower than that of bench-top ELISA. Aside from improving the sensitivity of paper-based ELISA, an important next step will be advancing paper-based ELISA into a portable device, where all the sample processing steps are performed automatically on the device. The combination of paper-based ELISA with new technologies for controlling and timing fluid flow in microPADs $[15,16]$ could lead to a paper-based version of POCKET and take paper-based devices out of the lab and into the real world.

\section{Naming the devices: it actually matters}

It is difficult to search the literature for articles on microPADs because a large percentage of 
journal articles start with the phrase, 'This paper describes...' or 'In this paper...', so when searching for articles on 'paper,' one inevitably gets hundreds of search results and must sort through them to identify the articles that are relevant. In order to minimize this problem, it is important for everyone working with microPADs to use common keywords to describe the devices. Aside from microPADs, the most common terms used to describe the devices have been paper-based microfluidic devices, paper-based diagnostic devices, patterned paper devices and 2D paper networks (2DPNs).

\section{Conclusion}

Four years after the initial publication on microPADs, the simple idea of making microfluidic devices out of paper is being adopted by a growing number of researchers. The argument for developing low-cost diagnostic devices is still as compelling as it was when the project started: millions of people die every year, mostly in developing countries, from preventable or treatable diseases [17] and portable diagnostic devices could help save many of these lives. MicroPADs have not yet made it into the field and have not yet saved any lives, but they are advancing steadily towards this goal.

While microPADs were first developed as diagnostic devices for use in developing countries, they could also be designed to detect a wide range of analytes, such as small molecules, antibodies, proteins and DNA, which would greatly expand the applications of the devices. MicroPADs could be used to monitor air, soil and water quality. They could be used as diagnostic devices for animals and plants, in home healthcare to diagnose disease or monitor drug levels or by the military and first-responders to assess a person's health status or detect toxins, biohazards or explosives. MicroPADs also have many potential applications in basic research. For example, they could provide a new platform for cell culture in biology [18], a new tool for studying capillary wicking in physics and a new platform for solid-phase synthesis of molecules in chemistry [19].

"MicroPADs could be used to monitor air, soil and water quality. They could be used as diagnostic devices for animals and plants, in home healthcare to diagnose disease or monitor drug levels or by the military and first-responders to assess a person's health status or detect toxins, biohazards or explosives."

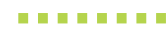

One of the great aspects of paper-based microfluidic devices is that there is a very low barrier to entry, both in terms of the cost of equipment and the technical expertise, which is required to fabricate devices. A pair of scissors and a paper towel is all that is really needed to make simple paper-based devices. So, much like open-source software, I look forward to seeing contributions to this field from all kinds of scientists from all over the world and seeing where we can go with this simple yet powerful technology.

\section{Financial \& competing interests disclosure \\ The author has no relevant affiliations or financial involve- ment with any organization or entity with a financial inter- est in or financial conflict with the subject matter or materi- als discussed in the manuscript. This includes employment, consultancies, honoraria, stock ownership or options, expert testimony, grants or patents received or pending, or royalties. \\ No writing assistance was utilized in the production of this manuscript.}

\section{References}

1 Sia SK, Linder V, Parviz BA et al. An integrated approach to a portable and low-cost immunoassay for resource-poor settings. Angew. Chem. Int. Ed. Engl. 43, 498-502 (2004).

2 Sia SK, Whitesides GM. Microfluidic devices fabricated in poly(dimethylsiloxane) for biological studies. Electrophoresis 24, 3563-3576 (2003)

3 von Lode P. Point-of-care immunotesting: approaching the analytical performance of central laboratory methods. Clin. Biochem. 38, 591-606 (2005).

4 Ellerbee AK, Phillips ST, Siegel AC et al. Quantifying colorimetric assays in paperbased microfluidic devices by measuring the transmission of light through paper. Anal. Chem. 81, 8447-8452 (2009).

5 Washburn EW. The dynamics of capillary flow. Phys. Rev. 17, 273-283 (1921).

6 Martinez AW, Phillips ST, Butte MJ et al. Patterned paper as a platform for inexpensive, low-volume, portable bioassays. Angew. Chem. Int. Ed. Engl. 46, 1318-1320 (2007).
7 Abe K, Suzuki K, Citterio D. Inkjet-printed microfluidic multianalyte chemical sensing paper. Anal. Chem. 80, 6928-6934 (2008).

8 Li X, Tian J, Nguyen T et al. Paper-based microfluidic devices by plasma treatment. Anal. Chem. 80, 9131-9134 (2008).

9 Olkkonen J, Lehtinen K, Erho T. Flexographically printed fluidic structures in paper. Anal. Chem. 82, 10246-10250 (2010).

10 Martinez AW, Phillips ST, Whitesides GM et al. Diagnostics for the developing world: microfluidic paper-based analytical devices. Anal. Chem. 82, 3-10 (2010). 


\section{EDITORIAL | Martinez}

11 Fenton EM, Mascarenas MR, López GP et al. Multiplex lateral-flow test strips fabricated by two-dimensional shaping. ACS Appl. Mater. Interfaces 1, 124-129 (2009).

12 Carrilho E, Martinez AW, Whitesides GM. Understanding wax printing: a simple micropatterning process for paper-based microfluidics. Anal. Chem. 81, 7091-7095 (2009).

13 Delaney JL, Hogan CF, Tian J et al. Electrogenerated chemiluminescence detection in paper-based microfluidic sensors. Anal. Chem. 83, 1300-1306 (2011).
14 Cheng CM, Martinez AW, Gong J et al. Paper-based ELISA. Angew. Chem. Int. Ed. Engl. 49, 4771-4774 (2010).

15 Noh H, Phillips ST. Fluidic timers for time dependent, point-of-care assays on paper. Anal. Chem. 82, 8071-8078 (2010).

16 Fu E, Lutz B, Kauffman P et al. Controlled reagent transport in disposable $2 \mathrm{D}$ paper networks. Lab. Chip 10, 917-920 (2010).

17 World Health Organization. The World Health Report 2005 - make every mother and child count (2005).
18 Derda R, Laromaine A, Mammoto A et al. Paper-supported 3D cell culture for tissue-based bioassays. Proc. Natl Acad. Sci. USA 106, 18457-18462 (2009).

19 Bowman MD, Jeske RC, Blackwell HE. Microwave-accelerated SPOT-synthesis on cellulose supports. Org. Lett. 6, 2019-2022 (2004). 